OPEN ACCESS

Edited by:

David Vauzour,

University of East Anglia,

United Kingdom

Reviewed by:

Chris Curran,

Northern Kentucky University,

United States

Ádám Nyúl-Tóth,

University of Oklahoma Health

Sciences Center, United States

*Correspondence:

Ryusuke Takechi

r.takechi@curtin.edu.au

Specialty section:

This article was submitted to

Nutrition and Brain Health,

a section of the journal

Frontiers in Nutrition

Received: 16 February 2021 Accepted: 08 April 2021

Published: 03 May 2021

Citation:

Graneri L, Lam V, D'Alonzo Z, Nesbit M, Mamo JCL and Takechi R (2021) The Consumption of Energy Drinks Induces Blood-Brain Barrier

Dysfunction in Wild-Type Mice.

Front. Nutr. 8:668514.

doi: 10.3389/fnut.2021.668514

\section{The Consumption of Energy Drinks Induces Blood-Brain Barrier Dysfunction in Wild-Type Mice}

\author{
Liam Graneri 1,2, Virginie Lam ${ }^{1,3}$, Zachary D'Alonzo ${ }^{1,2}$, Michael Nesbit ${ }^{1,2}$, \\ John C. L. Mamo ${ }^{1,2}$ and Ryusuke Takechi ${ }^{1,2 *}$
}

${ }^{1}$ Curtin Health Innovation Research Institute, Curtin University, Bentley, WA, Australia, ${ }^{2}$ Curtin Medical School, Faculty of Health Science, Curtin University, Bentley, WA, Australia, ${ }^{3}$ School of Population Health, Faculty of Health Science, Curtin University, Bentley, WA, Australia

Energy drinks containing significant quantities of caffeine and sugar are increasingly consumed, particularly by adolescents and young adults. Chronic ingestion of energy drinks may potentially regulate vascular risk factors. This study investigated the effects of chronic ingestion of energy drinks on blood-brain barrier (BBB) integrity and neuroinflammation. Male C57BL/6J mice were maintained on water (control), Mother ${ }^{\mathrm{TM}}$ (ED), sugar-free Mother ${ }^{\mathrm{TM}}$ (sfED), or Coca Cola ${ }^{\mathrm{TM}}$ soft drink (SD) for 13 weeks. The BBB integrity and neuroinflammation were analyzed with semi-quantitative immunofluorescent microscopy. Blood pressure, plasma inflammatory cytokine levels and blood glucose were also considered. Following 13 weeks of intervention, mice treated with ED, sfED, and SD showed significant disruption of BBB. However, marked neuroinflammation was observed only in sfED group mice. The consumption of ED and sfED significantly altered the blood pressure and plasma concentrations of inflammatory cytokines, TNF-a, IL-4, IL-6, and IL-10, and both increased plasma glucose. Correlation analyses showed significant associations between BBB dysfunction and hypotension, hyperglycaemia and cytokine dyshomeostasis. The intake of energy drink, particularly the sugar free formulation, may compromise the integrity of BBB and induce neuroinflammation via hypotension, hyperglycaemia and inflammatory pathways.

Keywords: energy drinks, metabolic syndrome, blood-brain barrier, neurovascular integrity, epithelial function

\section{INTRODUCTION}

The blood-brain barrier (BBB) describes the anatomical structure which surrounds the endothelial cells of cerebral capillaries (1). Its function is to serve as a selectively permeable barrier for brain parenchyme (2) and this is achieved ordinarily through highly regulated expression of endothelial tight junctions within the paracellular spaces. A disruption in BBB integrity permits the cerebral extravasation of plasma-borne molecules, which may activate astrocytes and microglia and promote neuroinflammation (3). Chronically exaggerated astrocytosis and microgliosis lead to heightened oxidative stress, which consequently promotes degeneration of neurons (4). Indeed, $\mathrm{BBB}$ dysfunction is commonly observed in various neurodegenerative disorders such as Alzheimer's disease and multiple sclerosis, suggesting a causal association (5-8).

Energy drinks are highly caffeinated, carbonated beverages first marketed in Europe and Asia in the 1960's (9). The primary ingredients with popular energy drinks such as Red 
Bull $^{\mathrm{TM}}$, Mother ${ }^{\mathrm{TM}}$, and Monster ${ }^{\mathrm{TM}}$ include caffeine/guarana [ 160 mg per serving $(375 \mathrm{ml})]$, remarkable abundance of taurine ( 2,000 mg per serving), B6/B12 vitamins ( $1.0 \mathrm{mg} / 0.5$ $\mu \mathrm{g}$ per serving, respectively), and in sugar-formulated energy drinks ( $\sim 51 \mathrm{~g}$ sucrose per serving). Recent population studies report significantly accelerating ingestion trends of energy drinks, particularly in young adults (10). Due to their complex formulations, both negative and positive effects on BBB integrity may be realized with regular, significant consumption of energy drinks. Evidence consistent with the latter are findings that energy drinks can have acute hypertensive effects on blood pressure, but paradoxically, hypotensive effects with chronic ingestion (11-17). Chronic consumption of sugar-formulated energy drinks may also have secondary indirect neurovascular effects, exacerbating hyperglycaemia, insulin resistance (18-22) and neurovascular integrity and neuroinflammation (23-25).

To gain better insight into the potential effects of energy drinks on BBB integrity, in this pilot study, genetically unmanipulated wild-type mice were chronically provided with diluted energy drinks as their sole drinking solution. The putative effects of Mother ${ }^{\mathrm{TM}}$, Sugar-free Mother ${ }^{\mathrm{TM}}$, and Coca-Cola ${ }^{\mathrm{TM}}$ (which lacks taurine, B-group vitamins and has substantially less caffeine) were studied in the context of neurovascular integrity and neuroinflammation.

\section{METHODS}

\section{Animals and Dietary Intervention}

Wild-type C57BL/6J male mice were purchased at 5 weeks of age from the Animal Resources Center (WA, Australia). After 1 week of acclimatization period, mice were randomly assigned to one of 4 drink intervention groups ( $n=10$ per group) for 13 weeks, each given a unique drink intervention. The control group was given only water. The drink groups were given either Mother ${ }^{\mathrm{TM}}$ energy drink (ED), sugar-free Mother ${ }^{\mathrm{TM}}$ (sfED), or Coca-Cola ${ }^{\mathrm{TM}}$ carbonated soft drink (SD); all diluted to $30 \%$ (v/v) in water. The age of mice and the study duration were determined based on previous studies where dietary intervention and cerebrovascular integrity were investigated $(7,26)$. The age of mice tested here is roughly equivalent to adolescent to young adult in human. The dose of each drink ingested was determined based on similar previous studies (27) in attempt to replicate the higher level of social relevance in the context of energy drink consumption. Male mice were chosen because energy drink consumption is more popular in men than women (28), and to be consistent with previous studies $(26,29)$. All groups were provided with standard rodent maintenance chow (AIN-93M, Specialty Feeds, WA, Australia). The major nutritional components of AIN93M chow, Mother ${ }^{\mathrm{TM}}$, sugar-free Mother ${ }^{\mathrm{TM}}$, and Coca-Cola ${ }^{\mathrm{TM}}$ are presented in Supplementary Table 1. The consumption of respective food and drink quantities were measured twice weekly. All animals were kept in individually ventilated cages with $12 \mathrm{~h}$ light/dark cycles, under controlled temperature $\left(21^{\circ} \mathrm{C}\right)$ and air pressure. All drinks and diets were available ad libitum. All animal protocols described in this study were approved by Curtin University Animal Ethics Committee (approval no. ARE2018-3).

\section{Blood Pressure Measurement}

At baseline and after 13 weeks of the drink intervention, systolic and diastolic blood pressures as well as mean arterial pressure were measured by using a CODA high-throughput tail cuff blood pressure system as described previously (30). Briefly, the mice were restrained, equipped with the tail cuff connected to CODA blood pressure reader and rested on an infrared heating pad. After 5 acclimatization trials, 15 readings of each blood pressure measure were taken with $10 \mathrm{~s}$ intervals between the measurements. The results are presented as a mean of all valid readings of the 15 measurements. The mean change of blood pressure from baseline to the 13 weeks per individual mouse is presented.

\section{Brain and Blood Sample Collection}

Following the blood pressure analysis, the mice were anesthetized with gaseous isoflurane and blood samples were collected via cardiac puncture. Whole blood was collected, allowed to clot at room temperature for $30 \mathrm{~min}$ and centrifuged for $10 \mathrm{~min}$ at 4,000 rpm. Thereafter, the supernatant serum was aliquoted and stored at $-80^{\circ} \mathrm{C}$ for further analysis. Each mouse brain was carefully removed, and the right hemisphere was fixed in $4 \%$ paraformaldehyde for $24 \mathrm{~h}$ and subsequently cryoprotected with $20 \%$ sucrose for $72 \mathrm{~h}$ before freezing in isopentane/dry ice and stored at $-80^{\circ} \mathrm{C}$ for immuno-microscopy analyses.

\section{Assessment of BBB Integrity}

The integrity of cerebral capillaries was assessed using an established protocol of blood-borne immunoglobulin-G (IgG) extravasation into the brain peri-vascular parenchymal space as an indication of non-specific "leakage" across the BBB (7, 26, 31). Briefly, $20 \mu \mathrm{m}$ thick cryosections were blocked with $10 \%$ goat serum in PBS for $30 \mathrm{~min}$ and subsequently incubated with goat anti-mouse IgG conjugated with Alexa488 fluorophore (1:200, ThermoFisher, USA) for $20 \mathrm{~h}$ in the dark at $4^{\circ} \mathrm{C}$. Thereafter, the nuclei were counterstained with DAPI (1:500) and mounted with antifade mounting media. The slides were viewed under a confocal microscope (UltraVIEW Vox, PerkinElmer). On average, 11 3-D images were captured, respectively, in the hippocampus and cortex of each brain section at $20 \times$ magnification to cover the majority of each brain region; and each 3 -D image consisted of $20 \mathrm{Z}$-slices with $1 \mu \mathrm{m} \mathrm{z}$-axis distance. The image capturing conditions (i.e., laser power, camera sensitivity and exposure time) were kept consistent amongst all animals of all experimental groups. The abundance of IgG extravasation was determined semi-quantitatively per image by measuring the voxel intensity of Alexa dye in the areas of peri-vascular parenchymal leakage using Volocity (PerkinElmer) as detailed previously (7). Briefly, the area of IgG extravasation (diffuse weak staining around the vessels) was selected with a magic wand tool of the software, which determines the voxel intensity of the selected region of interest. The experienced investigators identified the vessel area based on the intense IgG staining and endothelial nuclei staining and excluded these areas from the quantitation. The investigators were blinded to the treatment groups to eliminate any bias to these analyses. 


\section{Analyses of Neuroinflammation}

Neuroinflammation was assessed by determining ionized calcium-binding adapter molecule 1 (Iba-1) expression through quantitative immunofluorescent microscopy as established previously (32). Twenty $\mu \mathrm{m}$ sections underwent a $20 \mathrm{~h}$ antigen retrieval at $37^{\circ} \mathrm{C}$ in Tris-EDTA buffer. The sections were then rehydrated in PBS before being blocked in $10 \%$ goat serum in PBS for $30 \mathrm{~min}$, and subsequently incubated with rabbit antimouse Iba-1 (1:500, WAKO, USA) for $20 \mathrm{~h}$ at $4{ }^{\circ} \mathrm{C}$. The sections were then incubated with goat anti-rabbit IgG conjugated with Alexa 488 fluorophore (1:500, ThermoFisher) for $2 \mathrm{~h}$ in the dark at $20^{\circ} \mathrm{C}$. The slides were viewed and imaged with UltraVIEW Vox in the same manner as for the IgG staining protocol above. Fluorescence was quantitated with Volocity (ver 5.4.2) based on pre-set threshold parameters to ensure adequate overall fit for intensity threshold of all sections.

\section{Determination of Inflammatory Cytokines in Serum}

Serum concentrations of inflammatory cytokines interleukin (IL)-2, IL-4, IL-6, IL-10, and tumor necrosis factor (TNF)- $\alpha$ were determined using a cytometric bead assay by using a commercial kit (BD Biosciences MA, USA) as reported previously (33). Briefly, $10 \mu \mathrm{l}$ of undiluted serum, $10 \mu \mathrm{l}$ of capture beads mixture, and $10 \mu \mathrm{l}$ of PE detection reagent were mixed in a 96-well microplate and incubated in the dark for $2 \mathrm{~h}$. After a wash, and centrifugation, the beads were resuspended (7) in $200 \mu \mathrm{l}$ wash buffer. Results were acquired using FACS Canto II (BD Biosciences) and the data was analyzed by using FlowJo (FlowJo, OR, USA). The standards provided in the kit were used to construct a one-phase decay non-linear regression line that was subsequently used to interpolate the sample results.

\section{Statistical Methods}

Based on previous similar studies, power calculation was done with effect size $d=1.1, \alpha$ at 0.3 , and power $=0.9$, which indicated 10 mice per group to provide sufficient power (26). The data for each measure were inputted into GraphPad Prism (ver. 7.04 Graphpad Software, CA, US) and expressed as mean \pm SEM. Data normality was tested with D'Agostino-Pearson omnibus normality test. Statistical analysis was conducted using a one-way ANOVA for with Fisher's LSD post-hoc multiple comparison test for normally distributed data, whilst nonparametric Kruskal-Wallis test with Dunn's post-hoc test was used for the data without normal distribution. Statistical significance was determined at $p<0.05$. Associations between BBB dysfunction or neuroinflammation and blood pressure or plasma inflammatory cytokines were assessed with Pearson's correlation analysis for the data that are normally distributed. For the data that were not normally distributed, non-parametric Spearman's correlation coefficient analysis was used.

\section{RESULTS}

The putative effects of chronic ingestion of either ED or sfED on neurovascular integrity and neuroinflammation were determined in wild-type C57BL/6J male mice. Mice were provided ad-libitum with diluted ED or sfED that were physiologically relevant. Consumption of chow, drink and energy intake are provided in Supplementary Figures 1A,B. The drink interventions were well-tolerated, and no adverse events were indicated. A substantially larger ingestion of drinking solutions were indicated in ED, sfED and SD groups compared to mice provided with water. However, the cumulative energy intake over the intervention period was comparable between treatments, due to a compensatory decrease in chow consumption in $\mathrm{ED}$ and SD treated mice (Supplementary Figure 1C). At 13 weeks of intervention, the body weight of the mice maintained on ED, sfED and SD were comparable to the control (Supplementary Figure 1D). Mice maintained on ED and SD were hyperlgycemic, however surprisingly, a similar increase in blood glucose was also realized in the mice that received sfED for 13 weeks (Supplementary Figure 1E).

Cerebrovascular BBB permeability was assessed by determining brain parenchymal extravasation of IgG. Figure 1 and Supplementary Figure 2 depict IgG abundance within cortex (CTX) and hippocampal formation (HPF). Mice maintained on ED demonstrated twice the abundance of parenchymal IgG compared to control mice maintained on water alone, indicating significant breakdown of BBB. However, the amplification effect of ED treatment on parenchymal IgG was comparable to mice provided SD. Mice maintained on sfED also had exaggerated parenchymal IgG, although this effect was less than that realized in either ED or SD treated mice.

Neuroinflammation was assessed by quantitatively measuring Iba-1, a sensitive marker of microglial activation. Increased $\mathrm{BBB}$ permeability in $\mathrm{ED}$ and $\mathrm{SD}$ treated mice did not result in heightened neuroinflammation compared to the control mice (Figure 2 and Supplementary Figure 3). However, mice maintained on the sfED had markedly exaggerated microglial activation.

The effects of EDs and SD on blood pressure are provided in Figures 3A-C. The mice maintained on ED for 13 weeks showed decreasing trend in systolic blood pressure compared to the control mice receiving water (Figure 3A). The reduction of diastolic pressure and mean arterial pressure in $\mathrm{ED}$ mice was significant (Figures 3B,C). In sfED treated mice, there was a significant reduction of systolic pressure compared to the control mice. Similar significant reductions were indicated for diastolic pressure and mean arterial pressure compared to the control group. In SD mice, the blood pressure was comparable to the control mice.

The serum concentration of inflammatory cytokines, IL4, IL-10, and TNF- $\alpha$ were determine (Figures 4A-D). TNF- $\alpha$, a pro-inflammatory cytokine, was modestly increased in ED treated mice compared to control mice and no other significant differences were realized for IL-4, IL-6, or IL-10. The plasma inflammatory cytokine in SD group was also unremarkable. In sfED treated mice where inflammation was realized, TNF- $\alpha$ was not exaggerated but rather, sfED mice had lower levels of IL- 4 and IL-10 and elevated IL-6. 

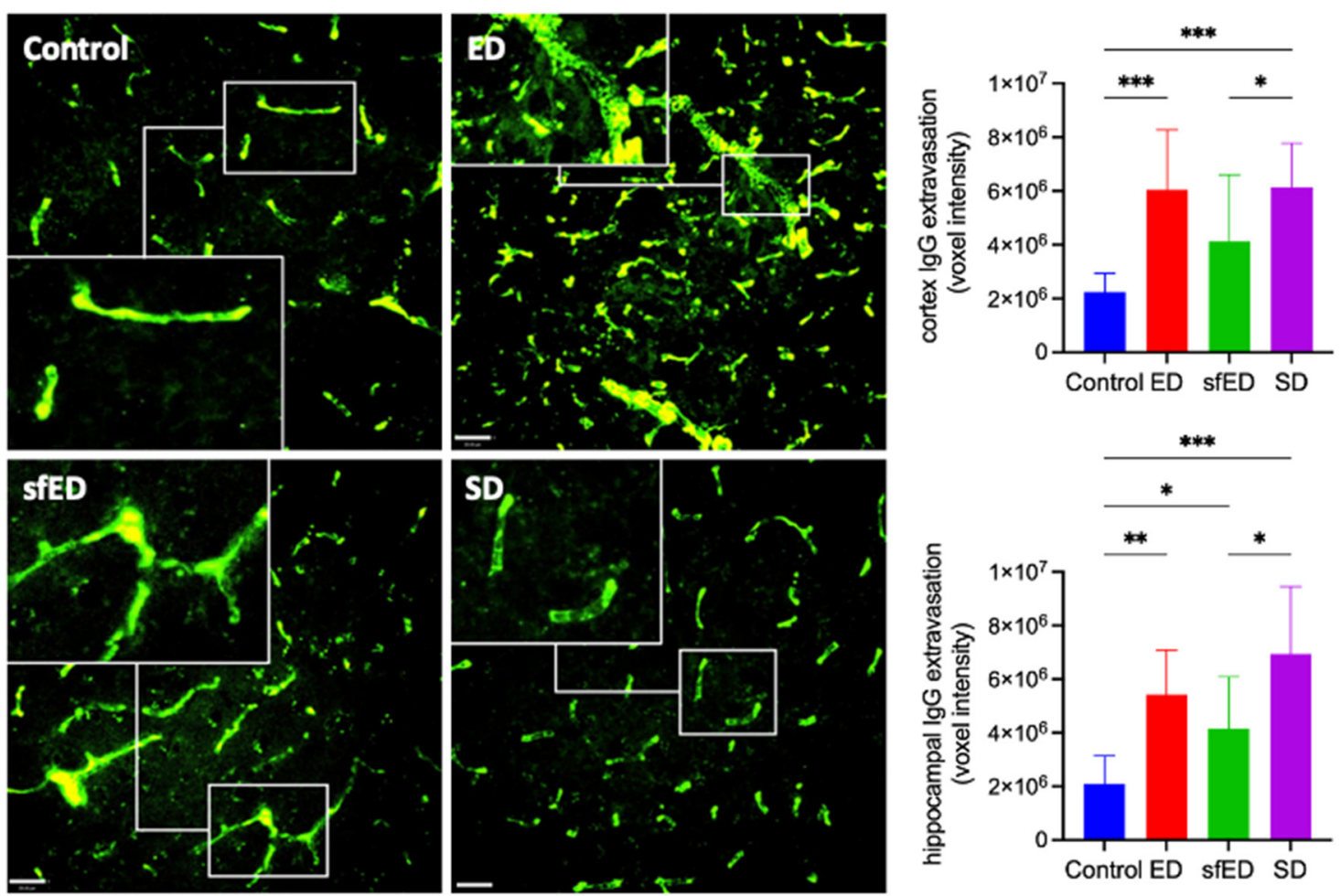

FIGURE 1 | Cerebrovascular integrity measures. Twenty $\mu \mathrm{m}$ sections of brain tissue for each treatment group were stained for lgG (green) and measured under a confocal fluorescent microscope. A representative image of cortex from each group is shown for both stains at low power magnification ( $20 \mathrm{x})$ (scale bar $=20 \mu \mathrm{m})$. The IgG images are accompanied by a high power magnified image (40x) in the white rectangular sub-frame within each low power image. All measures are of voxel intensity and therefore render the data to show relative levels rather than absolute concentrations. Statistical analysis was performed using Kruskal-Wallis test with uncorrected Dunn's post-hoc analysis ( $\left.n=10 ;{ }^{*} p<0.05,{ }^{* \star} p<0.005,{ }^{* \star *} p<0.0005\right)$.

\section{DISCUSSION}

Previous studies show detrimental effects of energy drink consumption on brain function. Diaz et al. demonstrated in rats, substantial inflammation and oxidative stress within the hippocampus and temporal cortex following the intake of energy drinks (34). Furthermore, the ingestion of energy drinks resulted in neurodegeneration and astrocytosis in hippocampal CA1 and dentate gyrus regions of adult rats (29). However, the mechanisms whereby the energy drink consumption compromise cerebral function and integrity are presently unknown. In the current study, the putative effects of chronic ingestion of energy drink, Mother ${ }^{\text {TM }}$ (ED) and sugar free Mother ${ }^{\mathrm{TM}}$ (sfED) in comparison to Coca-Cola ${ }^{\mathrm{TM}}$ (SD) on neurovascular integrity and neuroinflammation were determined in wild-type $\mathrm{C} 57 \mathrm{BL} / 6 \mathrm{~J}$ male mice. The higher energy intake through the ED or SD drink interventions was compensated by the lowered chow consumption, which resulted in the comparable total energy intake and weight gain across all intervention groups including the control group receiving only water. Nonetheless, the intake of ED, SD and indeed sfED led to a significant increase in blood glucose.

Following the 13 week drink interventions, this study demonstrated substantially greater BBB permeability in the mice that were maintained on ED, compared to the water-fed control mice. However, the exaggerated parenchymal abundance of IgG in $\mathrm{ED}$ mice was comparable to $\mathrm{SD}$ group mice. The latter observation suggests that the combination of taurine, B-group vitamins and significantly greater caffeine in the $\mathrm{ED}$ beverage does not exacerbate the BBB dysfunction induced by an ingestion of SD per se. Nonetheless, the experimental design adopted in this study cannot rule antagonistic components of ED differentially modulating $\mathrm{BBB}$ function. The sfED treated mice also had exaggerated parenchymal IgG, although less substantial than ED or SD. The data suggest that the provision of significant amounts of sucrose in $\mathrm{ED}$ and $\mathrm{SD}$ beverages contribute in part to the disruption of cerebral capillary BBB integrity.

Increased $\mathrm{BBB}$ permeability indicated in $\mathrm{ED}$ and SD treated mice did not result in neuroinflammation as indicated by the surrogate marker, Iba- 1 . The observation suggests that the degree of $\mathrm{BBB}$ dysfunction is modest and insufficient to activate astroglial cells. Rather, mice provided with sfED had marked microglial activation. One potential mechanism for the differential effects of ED vs. sfED is that the provision of the artificial sweetener including sucralose and acesulfame potassium is pro-inflammatory, or as a consequence of interactive effects with macro/micronutrients of the energy drink. The sfED formulation may also have had secondary indirect effects 

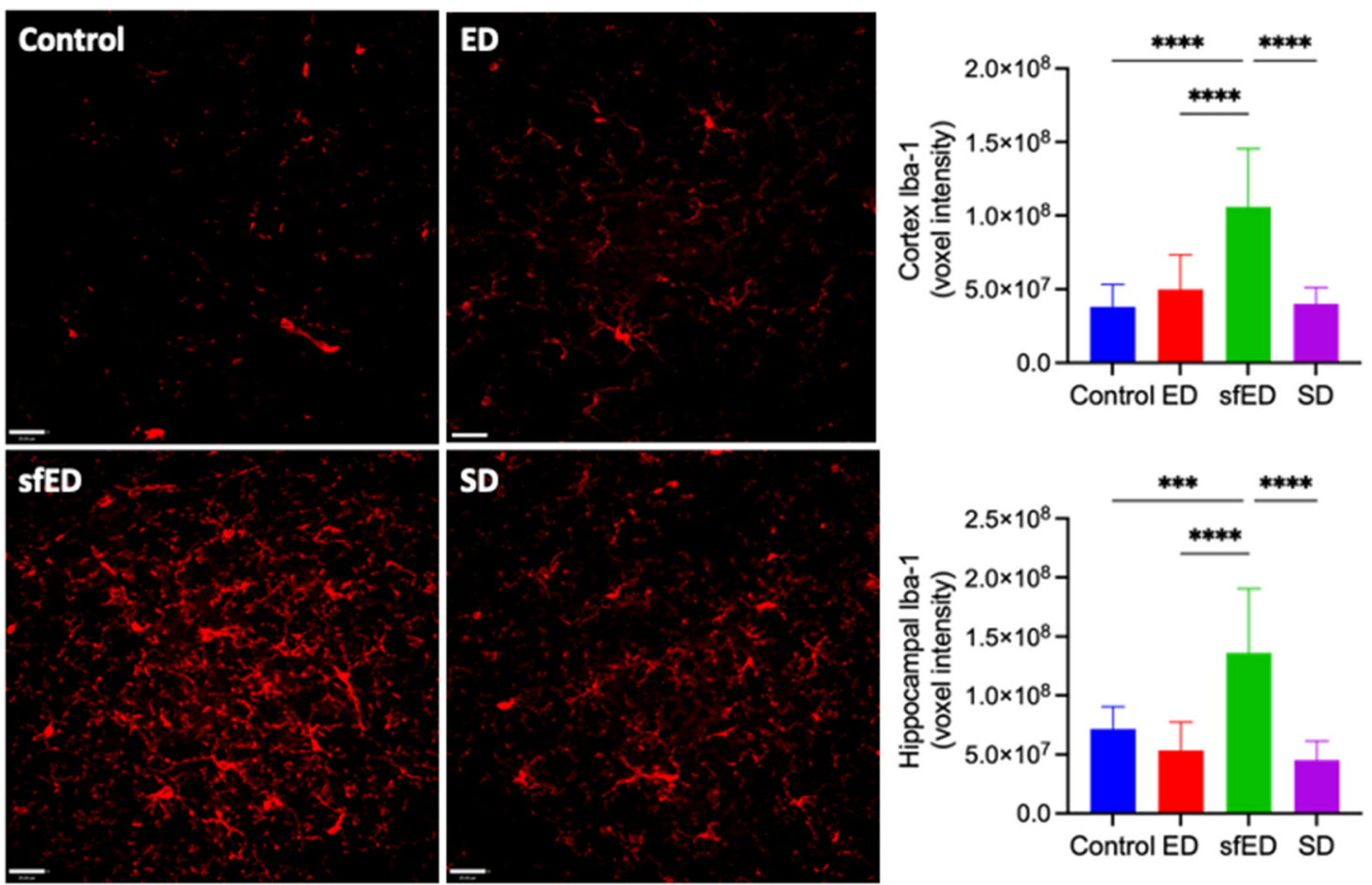

FIGURE 2 | Neuroinflammatory measures. Twenty $\mu \mathrm{m}$ sections of brain tissue for each treatment group were stained for lba-1 (red) and measured under a confocal fluorescent microscope. A representative image of each group is shown for both stains at low power magnification (20x) (scale bar $=20 \mu \mathrm{m})$. All measures are of voxel intensity and therefore render the data to show relative levels rather than absolute concentrations. Statistical analysis was performed using one way ANOVA with Fisher's LSD post-hoc analysis $\left(n=10 ;{ }^{* \star \star} p<0.0005 ;{ }^{* \star \star \star} p<0.0001\right)$
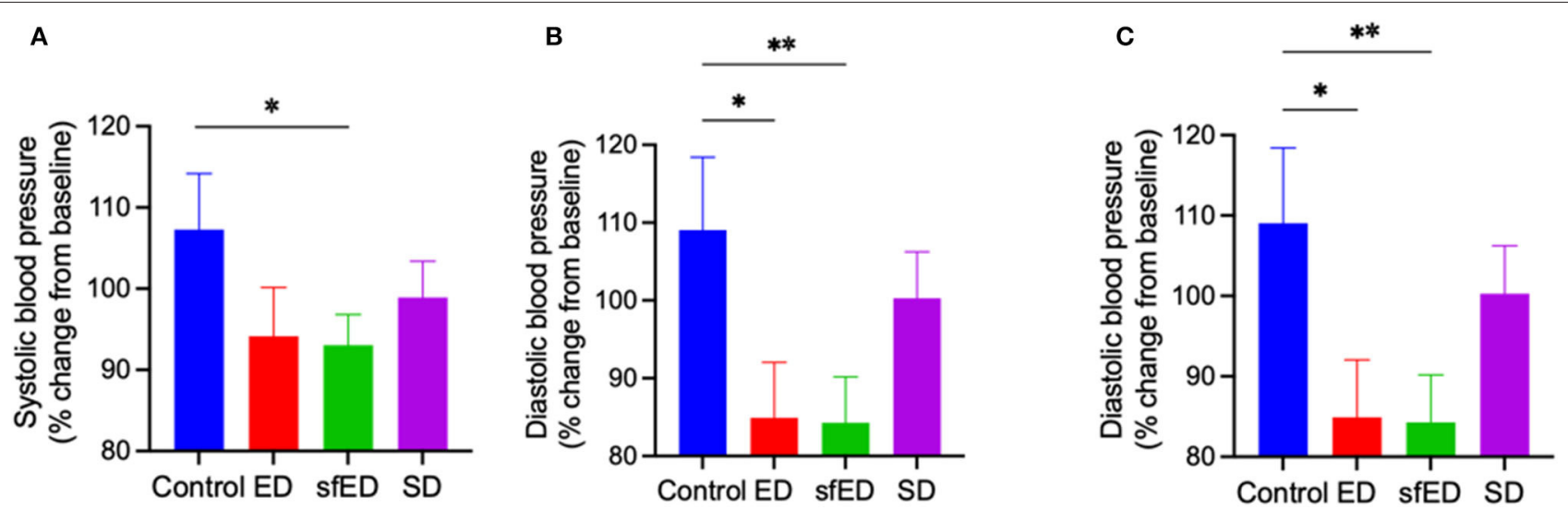

FIGURE 3 | Blood pressure. The mice received Mother (ED), sugar-free Mother (sfED), Coca-Cola (SD), or water (Control) for 13 weeks. The change in systolic blood pressure (A), diastolic blood pressure (B), and mean arterial pressure (C) between baseline and at the end of the experiment (13 weeks) were measured using a tail cuff method. Statistical analysis was performed using a one-way ANOVA with Fisher's LSD post-hoc analysis $\left(n=10 ;{ }^{*} p<0.05,{ }^{* *} p<0.005\right)$.

on neuroinflammation, via modulation of other physiological parameters. Consistent with the latter, we confirmed that chronic ingestion of ED markedly reduced blood pressures, consistent with our previous findings (17). Whilst caffeine is widely recognized to raise blood pressure, taurine has marked acute and chronic hypotensive effects (35-37). Ishikawa et al. showed that drug-induced hypotension in dogs resulted in BBB dysfunction
(38). Similarly, in a rabbit model of hyper- and hypo-tension the effects on BBB integrity were found to be U-shaped, but with hypotension effects being significantly greater than the hypertension-induced BBB hyperpermeability (39).

Key specific cytokines were assessed in the context of modulation of cerebrovascular BBB integrity and neuroinflammation. Interestingly, a modest but nonetheless 


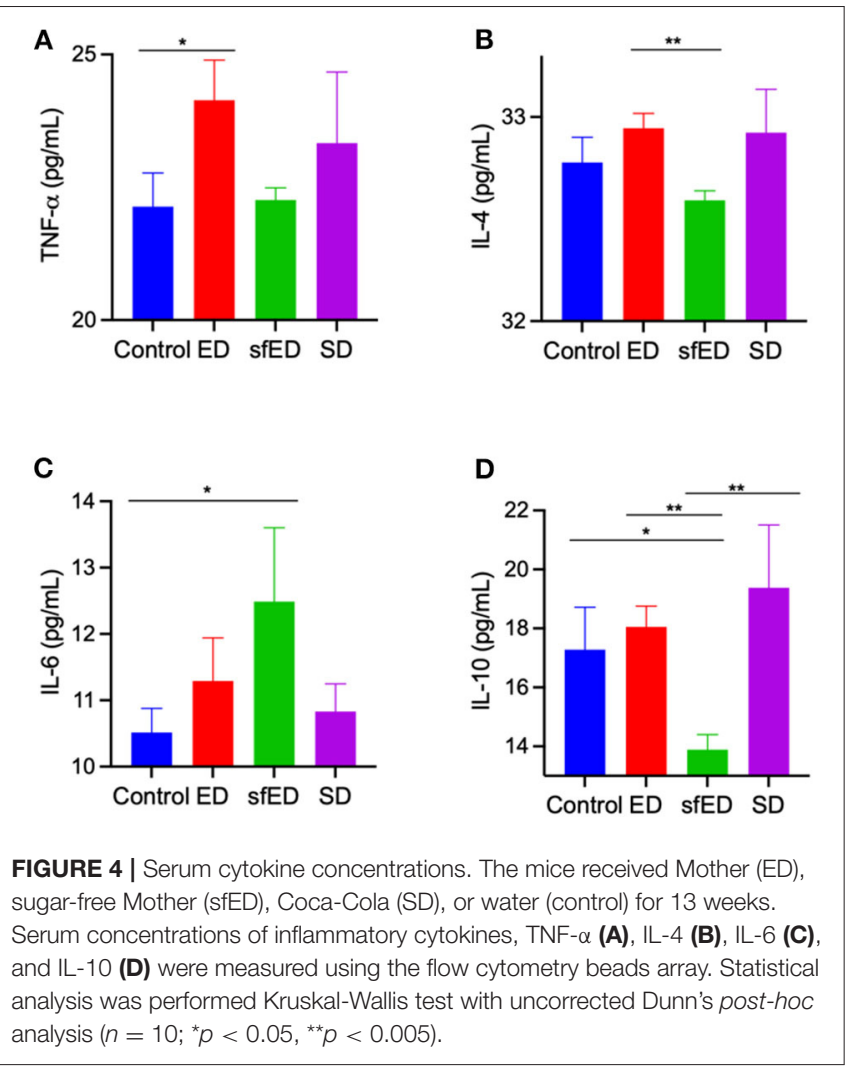

significant increase in TNF- $\alpha$ was realized in $\mathrm{ED}$ and to a lesser extent in SD treated mice. This may be due to AGERAGE interaction which occurs in hyperglycaemic conditions, consequently releasing TNF- $\alpha$ and inducing neuroinflmmation and oxidative stress in diabetic neuropathy (25). However, in this species with the indicated intervention with $\mathrm{ED}$ or $\mathrm{SD}$, this was not associated with neuroinflammation per se. Compensatory changes in anti-inflammatory IL-4 or IL-10 were not suggested in $\mathrm{ED}$ or $\mathrm{SD}$, with plasma concentrations comparable to control mice provided water. Rather in sfED mice where marked glial cell activation was indicated, there was modestly greater plasma abundance of the pro-inflammatory cytokine, IL- 6 and reduced levels of the anti-inflammatory IL- 4 and particularly IL-10. This marked reduction of anti-inflammatory cytokines may be induced by artificial sweeteners as reported previously (40). Therefore, in sfED, neuroinflammation may in part have reflected a shift to a more inflammatory plasma cytokine phenotype.

In conclusion, in a rodent model the long-term intake of ED (Mother ${ }^{\mathrm{TM}}$ ) increased capillary permeability, but was equipotent to $\mathrm{SD}\left(\mathrm{Coca}-\mathrm{Cola}^{\mathrm{TM}}\right)$. Consumption of $\mathrm{ED}$ or $\mathrm{SD}$ had no

\section{REFERENCES}

1. Abbott NJ. Dynamics of CNS barriers: evolution, differentiation, and modulation. Cell Mol Neurobiol. (2005) 25:523. doi: 10.1007/s10571-004-1374-y

2. Abbott NJ, Patabendige AAK, Dolman DEM, Yusof SR, Begley DJ. Structure and function of the blood-brain barrier. significant effect on measures of neuroinflammation, despite some indication of modestly exaggerated pro-inflammatory cytokines. Moreover, elevation of blood pressures was not indicated, despite significant intake of caffeine. Rather, consumption of sfED induced marked microglial activation, concomitant with a substantial reduction in blood pressure and a modest change in plasma cytokines toward a more pro-inflammatory state. The differential effects of sfED and ED suggest that the artificial sweetener may be causally associated with the detrimental neuroinflammatory effects realized. However, the present study was a preliminary study and did not examine the effects of specific individual component of energy drinks on BBB. In addition, the present study did not consider sex differences. Therefore, future studies may consider these points and may attempt to identify the macro/micronutrient that is responsible for the disruption of $\mathrm{BBB}$ and increased neuroinflammation.

\section{DATA AVAILABILITY STATEMENT}

The original contributions presented in the study are included in the article/Supplementary Material, further inquiries can be directed to the corresponding author/s.

\section{ETHICS STATEMENT}

The animal study was reviewed and approved by Curtin Animal Ethics Committee.

\section{AUTHOR CONTRIBUTIONS}

The study was designed by LG, VL, JM, and RT. The experiments were carried out by LG, VL, ZD'A, MN, and RT. The data collection and interpretation were done by LG, VL, ZD'A, MN, JM, and RT. The manuscript was prepared by LG, VL, MN, JM, and RT. All authors contributed to the article and approved the submitted version.

\section{FUNDING}

This study was financially supported by Western Australian Department of Health and National Health and Medical Research Council of Australia.

\section{SUPPLEMENTARY MATERIAL}

The Supplementary Material for this article can be found online at: https://www.frontiersin.org/articles/10.3389/fnut.2021. 668514/full\#supplementary-material 
feature of neurodegenerative disorders. Front Pharmacol. (2019) 10:1008. doi: 10.3389/fphar.2019.01008

5. Zlokovic BV. Neurovascular pathways to neurodegeneration in Alzheimer's disease and other disorders. Nat Rev Neurosci. (2011) 12:723-38. doi: 10.1038/nrn3114

6. Sweeney MD, Sagare AP, Zlokovic BV. Blood-brain barrier breakdown in Alzheimer disease and other neurodegenerative disorders. Nat Rev Neurol. (2018) 14:133-50. doi: 10.1038/nrneurol.2017.188

7. Takechi R, Lam V, Brook E, Giles C, Fimognari N, Mooranian A, et al. Bloodbrain barrier dysfunction precedes cognitive decline and neurodegeneration in diabetic insulin resistant mouse model: an implication for causal link. Front Aging Neurosci. (2017) 9:399. doi: 10.3389/fnagi.2017.00399

8. Nation DA, Sweeney MD, Montagne A, Sagare AP, D’Orazio LM, Pachicano $M$, et al. Blood-brain barrier breakdown is an early biomarker of human cognitive dysfunction. Nat Med. (2019) 25:270-6. doi: 10.1038/s41591-018-0297-y

9. Finnegan D. The health effects of stimulant drinks. Nutri Bull. (2003) 28:14755. doi: 10.1046/j.1467-3010.2003.00345.x

10. Trapp GSA, Allen KL, O'Sullivan T, Robinson M, Jacoby P, Oddy WH. Energy drink consumption among young Australian adults: associations with alcohol and illicit drug use. Drug Alcohol Depend. (2014) 134:307. doi: 10.1016/j.drugalcdep.2013.09.006

11. Daly JW, Butts-Lamb P, Padgett W. Subclasses of adenosine receptors in the central nervous system: interaction with caffeine and related methylxanthines. Cell Mol Neurobiol. (1983) 3:69-80. doi: 10.1007/BF00734999

12. Schaffer $S$, Lombardini $B$, Azuma J. Interaction between the actions of taurine and angiotensin II. Amino Acids. (2000) 18:305-18. doi: 10.1007/PL00010320

13. Yu H, Yang $T$, Gao $P$, Wei $X$, Zhang H, Xiong $S$, et al. Caffeine intake antagonizes salt sensitive hypertension through improvement of renal sodium handling. Sci Rep. (2016) 6:25746. doi: 10.1038/srep25746

14. Hartley TR, Lovallo WR, Whitsett TL, Sung BH, Wilson MF. Caffeine and stress: implications for risk, assessment, and management of hypertension. $J$ Clin Hyperten. (2007) 3:354-82. doi: 10.1111/j.1524-6175.2001.00478.x

15. Hartley TR, Sung BH, Pincomb GA, Whitsett TL, Wilson MF, Lovallo WR. Hypertension risk status and effect of caffeine on blood pressure. Hypertension. (2000) 36:137-41. doi: 10.1161/01.HYP.36.1.137

16. Umemura T, Ueda K, Nishioka K, Hidaka T, Takemoto H, Nakamura S, et al. Effects of acute administration of caffeine on vascular function. Am J Cardiol. (2006) 98:1538-41. doi: 10.1016/j.amjcard.2006.06.058

17. Graneri L, D’Alonzo Z, Lam V, Mamo J, Dhaliwal S, Takechi R. Chronic consumption of a commercial energy drink reduces blood pressure in normotensive wild-type mice. Front Nutr. (2019) 6:111. doi: 10.3389/fnut.2019.00111

18. Bian X, Chi L, Gao B, Tu P, Ru H, Lu K. The artificial sweetener acesulfame potassium affects the gut microbiome and body weight gain in CD-1 mice. PLoS ONE. (2017) 12:e0178426. doi: 10.1371/journal.pone.0178426

19. Elliott SS, Keim NL, Stern JS, Teff K, Havel PJ. Fructose, weight gain, and the insulin resistance syndrome. Am J Clin Nutri. (2002) 76:91122. doi: $10.1093 / \mathrm{ajcn} / 76.5 .911$

20. Keijzers GB, De Galan BE, Tack CJ, Smits P. Caffeine can decrease insulin sensitivity in humans. Diabetes Care. (2002) 25:364-9. doi: 10.2337/diacare.25.2.364

21. Malaisse WJ, Vanonderbergen A, Louchami K, Jijakli H, MalaisseLagae F. Effects of artificial sweeteners on insulin release and cationic fluxes in rat pancreatic islets. Cell Signal. (1998) 10:727-33. doi: 10.1016/S0898-6568(98)00017-5

22. Sarkar P, Basak P, Ghosh S, Kundu M, Sil PC. Prophylactic role of taurine and its derivatives against diabetes mellitus and its related complications. Food Chem Toxicol. (2017) 110:109-21. doi: 10.1016/j.fct.2017. 10.022

23. Farouque HMO, O'Brien RC, Meredith IT. Diabetes mellitus and coronary heart disease - from prevention to intervention: Part I. Austr N Zeal J Med. (2008) 30:351-9. doi: 10.1111/j.1445-5994.2000.tb00837.x

24. Goh S-Y, Cooper ME. The role of advanced glycation end products in progression and complications of diabetes. J Clin Endocrinol Metabo. (2008) 93:1143-52. doi: 10.1210/jc.2007-1817

25. Sandireddy R, Yerra VG, Areti A, Komirishetty P, Kumar A. Neuroinflammation and oxidative stress in diabetic neuropathy: futuristic strategies based on these targets. Int J Endocrinol. (2014) 2014:674987. doi: 10.1155/2014/674987

26. Takechi R, Pallebage-Gamarallage MM, Lam V, Giles C, Mamo JC. Nutraceutical agents with anti-inflammatory properties prevent dietary saturated-fat induced disturbances in blood-brain barrier function in wildtype mice. J Neuroinflamm. (2013) 10:73. doi: 10.1186/1742-2094-10-73

27. Ferreira SE, Hartmann Quadros IM, Trindade ÁA, Takahashi S, Koyama RG, Souza-Formigoni MLO. Can energy drinks reduce the depressor effect of ethanol? An experimental study in mice. Physiol Behav. (2004) 82:8417. doi: 10.1016/S0031-9384(04)00284-7

28. Friis K, Lyng JI, Lasgaard M, Larsen FB. Energy drink consumption and the relation to socio-demographic factors and health behaviour among young adults in Denmark. A population-based study. Eur J Public Health. (2014) 24:840-4. doi: 10.1093/eurpub/cku003

29. Kamar SA, Malak HWA, Saad SA. Effect of caffeinated energy drinks on the structure of hippocampal cornu ammonis 1 and dentate gyrus of adult male albino rats. Anatomy Cell Biol. (2020) 53:330-41. doi: 10.5115/acb.20.136

30. Mamo JCL, Lam V, Giles C, Coulson SH, Fimognari N, Mooranian A, et al. Antihypertensive agents do not prevent blood-brain barrier dysfunction and cognitive deficits in dietary-induced obese mice. Int J Obesity. (2017) 41:926. doi: 10.1038/ijo.2017.57

31. Takechi R, Pallebage-Gamarallage MM, Lam V, Giles C, Mamo JC. Longterm probucol therapy continues to suppress markers of neurovascular inflammation in a dietary induced model of cerebral capillary dysfunction. Lipids Health Dis. (2014) 13:91. doi: 10.1186/1476-511X-13-91

32. Tischer J, Krueger M, Mueller W, Staszewski O, Prinz M, Streit WJ, et al. Inhomogeneous distribution of Iba-1 characterizes microglial pathology in Alzheimer's disease. Glia. (2016) 64:1562-72. doi: 10.1002/glia.23024

33. Elahy M, Jackaman C, Mamo JC, Lam V, Dhaliwal SS, Giles C, et al. Bloodbrain barrier dysfunction developed during normal aging is associated with inflammation and loss of tight junctions but not with leukocyte recruitment. Immun Ageing. (2015) 12:2. doi: 10.1186/s12979-015-0029-9

34. Diaz A, Trevino S, Guevara J, Munoz-Arenas G, Brambila E, Espinosa B, et al. Energy drink administration in combination with alcohol causes an inflammatory response and oxidative stress in the hippocampus and temporal cortex of rats. Oxid Med Cell Longev. (2016) 2016:8725354. doi: 10.1155/2016/8725354

35. Mensegue MF, Burgueno AL, Tellechea ML. Perinatal taurine exerts a hypotensive effect in male spontaneously hypertensive rats and downregulates endothelial oxide nitric synthase in the aortic arch. Clin Exp Pharmacol Physiol. (2020) 47:780-9. doi: 10.1111/1440-1681.13260

36. Ibrahim MA, Eraqi MM, Alfaiz FA. Therapeutic role of taurine as antioxidant in reducing hypertension risks in rats. Heliyon. (2020) 6:e03209. doi: 10.1016/j.heliyon.2020.e03209

37. Guan L, Miao P. The effects of taurine supplementation on obesity, blood pressure and lipid profile: a meta-analysis of randomized controlled trials. Eur J Pharmacol. (2020) 885:173533. doi: 10.1016/j.ejphar.2020.173533

38. Ishikawa T, Funatsu N, Okamoto K, Takeshita H, McDowall DG. Bloodbrain barrier function following drug-induced hypotension in the dog. Anesthesiology. (1983) 59:526-31. doi: 10.1097/00000542-198312000-00007

39. Cook PR, Myers D, Barker M, Jones JG. Blood-brain barrier to pertechnetate following drug-induced hypotension. Br J Anaesth. (1989) 62:402-8. doi: 10.1093/bja/62.4.402

40. Rahiman F, Pool EJ. The in vitro effects of artificial and natural sweeteners on the immune system using whole blood culture assays. J Immunoassay Immunochem. (2014) 35:26-36. doi: 10.1080/15321819.2013.784197

Conflict of Interest: The authors declare that the research was conducted in the absence of any commercial or financial relationships that could be construed as a potential conflict of interest.

Copyright (c) 2021 Graneri, Lam, D'Alonzo, Nesbit, Mamo and Takechi. This is an open-access article distributed under the terms of the Creative Commons Attribution License (CC BY). The use, distribution or reproduction in other forums is permitted, provided the original author(s) and the copyright owner(s) are credited and that the original publication in this journal is cited, in accordance with accepted academic practice. No use, distribution or reproduction is permitted which does not comply with these terms. 